\title{
Local Quantum Measurement and No-Signaling Imply Quantum Correlations
}

\author{
H. Barnum, ${ }^{1}$ S. Beigi, ${ }^{2}$ S. Boixo, ${ }^{2, *}$ M. B. Elliott, ${ }^{2}$ and S. Wehner ${ }^{2}$ \\ ${ }^{1}$ Perimeter Institute for Theoretical Physics, 31 Caroline Street N, Waterloo, Ontario, N2L 2 Y5 Canada \\ ${ }^{2}$ Institute for Quantum Information, California Institute of Technology, Pasadena, California 91125, USA
}

(Received 26 November 2009; published 6 April 2010)

\begin{abstract}
We show that, assuming that quantum mechanics holds locally, the finite speed of information is the principle that limits all possible correlations between distant parties to be quantum mechanical as well. Local quantum mechanics means that a Hilbert space is assigned to each party, and then all local positiveoperator-valued measurements are (in principle) available; however, the joint system is not necessarily described by a Hilbert space. In particular, we do not assume the tensor product formalism between the joint systems. Our result shows that if any experiment would give nonlocal correlations beyond quantum mechanics, quantum theory would be invalidated even locally.
\end{abstract}

PACS numbers: 03.65.Ta, 03.65.Ud, 03.67.-a

Quantum correlations between spacelike separated systems are, in the words of Schrödinger, "the characteristic trait of quantum mechanics, the one that enforces its entire departure from classical lines of thought"[1]. Indeed, the increasing experimental support [2] for correlations violating Bell inequalities [3] is at odds with local realism. Quantum correlations have been investigated with increasing success [4], but what is the principle that limits them [5]?

Consider two experimenters, Alice and Bob, at two distant locations. They share a preparation of a bipartite physical system, on which they locally perform one of several measurements. This shared preparation may thereby cause the distribution over the possible two outcomes to be correlated. In nature, such nonlocal correlations cannot be arbitrary. For example, it is a consequence of relativity that information cannot propagate faster than light. The existence of a finite upper bound on the speed of information is known as the principle of no-signaling. This principle implies that if the events corresponding to Alice's and Bob's measurements are separated by spacelike intervals, then Alice cannot send information to Bob by just choosing a particular measurement setting. Equivalently, the probability distribution over possible outcomes on Bob's side cannot depend on Alice's choice of measurement setting, and vice versa. Quantum mechanics, like all modern physical theories, obeys the principle of nosignaling.

But is no-signaling the only limitation for correlations observed in nature? Bell [3] initiated the study of these limitations based on inequalities, such as the ClauserHorne-Shimony-Holt (CHSH) expression [6]. It is convenient to describe this inequality in terms of a game played by Alice and Bob. Suppose we choose two bits $x, y \in\{0,1\}$ uniformly and independently at random, and hand them to Alice and Bob, respectively. We say that the players win if they are able to return answers $a, b \in\{0,1\}$, respectively, such that $x y=a+b \bmod 2$. Alice and Bob can agree on any strategy beforehand, that is, they can choose to share any preparation possible in a physical theory, and choose any measurements in that theory, but there is no further exchange of information during the game. The probability that the players win is

$$
\frac{1}{4} \sum_{x, y \in\{0,1\}} \sum_{\substack{a, b \in\{0,1\} \\ x y=a+b \bmod 2}} p\left(a, b \mid M_{A}^{x}, M_{B}^{y}\right),
$$

where $p\left(a, b \mid M_{A}^{x}, M_{B}^{y}\right)$ denotes the probability that Alice and Bob obtain measurement outcomes $a$ and $b$ when performing the measurements $M_{A}^{x}$ and $M_{B}^{y}$, respectively (any preprocessing or postprocessing can be taken as part of the measurement operation). Classically, i.e., in any local realistic theory, this probability is bounded by [6]

$$
p_{\text {classical }} \leq 3 / 4 \text {. }
$$

Such an upper bound is called a Bell inequality.

Crucially, Alice and Bob can violate this inequality using quantum mechanics [3]. The corresponding bound is [7]

$$
p_{\text {quantum }} \leq \frac{1}{2}+\frac{1}{2 \sqrt{2}},
$$

and there exists a shared quantum state and measurements that achieve it [6]. Further, there is now compelling experimental evidence that nature violates Bell inequalities and does not admit a local realistic description [2]. Yet, there exist stronger no-signaling correlations (outside quantum mechanics) which achieve success probability $p_{\text {no-signal }}=$ 1 [5]. So why, then, is nature not more nonlocal [8]?

Studying limitations on nonlocal correlations thus forms an essential element of understanding nature. On one hand, it provides a systematic method to both theoretically and experimentally compare candidate physical theories [9]. On the other hand, it crucially affects our understanding of information in different settings such as cryptography and communication complexity [10-13]. For example, if na- 
ture would admit $p_{\text {no-signal }}=1$, any two-party communication problem could be solved using only a single bit of communication, independent of its size [11]. Also, for the special case of the CHSH inequality, it is known that the bound (3) is a consequence of information theoretic constraints such as uncertainty relations [12] or the recently proposed principle of information causality [13]. However, characterizing general correlations remains a difficult challenge [14], and it is interesting to consider what other constraints may impose limits on quantum correlations.

Result.-We forge a fundamental link between local quantum theory and nonlocal quantum correlations. In particular, we show that if Alice and Bob are locally quantum, then relativity theory implies that their nonlocal correlations admit a quantum description. The assumption of being locally quantum may thus provide another "reason" why the correlations we observe in nature are restricted by more than the principle of no-signaling itself. Figure 1 states our result.

Let us explain more formally what we mean by being locally quantum (see also Fig. 1). We say that Alice is locally quantum if her physical system can be described by means of a Hilbert space $\mathcal{H}_{A}$ of some fixed finite dimension $d$, on which she can perform any local quantum measurement [positive-operator-valued measurement $(\mathrm{POVM})] M_{A}=\left\{Q_{a}\right\}_{a}$ given by bounded operators $Q_{a} \in$ $\mathcal{B}\left(\mathcal{H}_{A}\right)$. The probability $p\left(a \mid M_{A}\right)$ that she obtains an outcome $a$ for measurement $M_{A}=\left\{Q_{a}\right\}_{a}$ is given by a function $\mathcal{B}\left(\mathcal{H}_{A}\right) \rightarrow[0,1]$ applied to the POVM elements. Gleason's theorem for POVM elements implies that the state of Alice's system is then described by a state $\rho_{A} \in$ $\mathcal{B}\left(\mathcal{H}_{A}\right)$ [15], and similarly for Bob, where we use $\mathcal{H}_{B}$ and $M_{B}=\left\{R_{b}\right\}_{b}$ to denote his Hilbert space and measurements, respectively. Conceptually, this means that quantum mechanics describes Alice and Bob's local physical systems.

However, we make no a priori assumption about the nature of the joint system held by Alice and Bob. In particular, we do not assume that it is described by a tensor product of their local Hilbert spaces, or that their joint system is quantum mechanical. This means that Alice and Bob can share any possible preparation which assigns probabilities to local POVM measurements. That is, their preparation is simply a function $\omega$ such that the probabilities of observing outcomes $a$ and $b$ for measurements $M_{A}=\left\{Q_{a}\right\}_{a}$ and $M_{B}=\left\{R_{b}\right\}_{b}$ are given by $p\left(a, b \mid M_{A}, M_{B}\right)=\omega\left(Q_{a}, R_{b}\right)$. In particular, the state of their joint system may not be described by any density matrix.

Nevertheless, we are able to show that just from the assumptions that Alice and Bob are locally quantum and that the no-signaling principle is obeyed, it follows that there exists a Hilbert space $\mathcal{H}_{A B}=\mathcal{H}_{A} \otimes \mathcal{H}_{B}$, a state $\rho_{A B} \in \mathcal{B}\left(\mathcal{H}_{A B}\right)$, and measurements $\tilde{M}_{A}=\left\{\tilde{Q}_{a}\right\}_{a}$ and $\tilde{M}_{B}=\left\{\tilde{R}_{b}\right\}_{b}$ for Alice and Bob, such that

$$
p\left(a, b \mid M_{A}, M_{B}\right)=\omega\left(Q_{a}, R_{b}\right)=\operatorname{tr}\left(\left(\tilde{Q}_{a} \otimes \tilde{R}_{b}\right) \rho_{A B}\right) .
$$

That is, all correlations can be reproduced quantum mechanically.

Implications.-Our result solves an important piece of the puzzle of understanding nonlocal correlations, and their relation to the rich local phenomena we encounter in quantum theory such as Bohr's complementarity principle, Heisenberg uncertainty, and Kochen-Specker noncontextuality. In particular, it implies that if we obey local quantum statistics we can never hope to surpass a Tsirelson-type bound on $p_{\text {quantum }}$ like that of (3), ruling out the possibility of such striking differences with respect
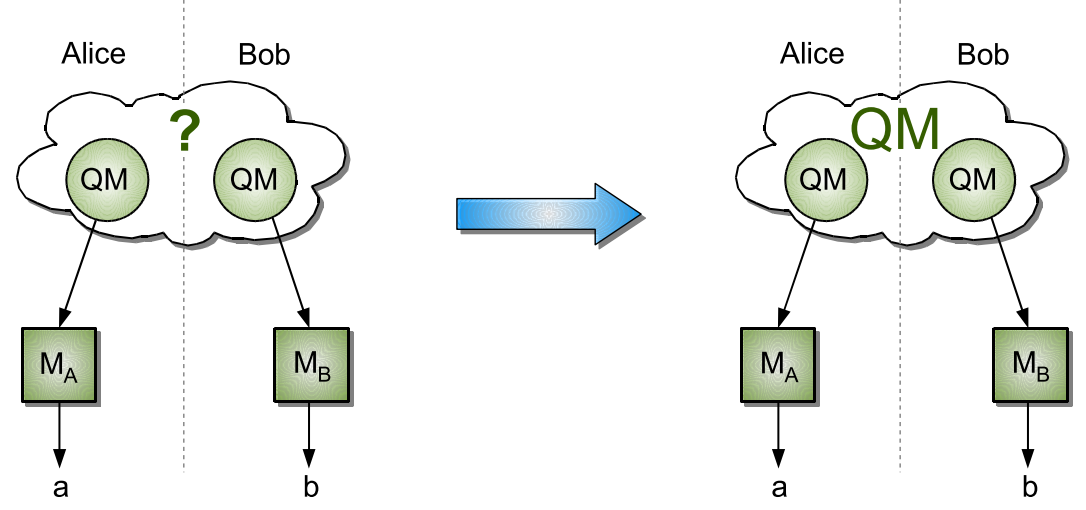

FIG. 1 (color online). If the principle of no-signaling is obeyed and Alice and Bob are locally quantum, their nonlocal correlations can be obtained in quantum mechanics. Alice and Bob are locally quantum if their local systems can be described by a Hilbert space and they can choose to measure any local POVM $M_{A}=\left\{Q_{a}\right\}_{a}$ and $M_{B}=\left\{R_{b}\right\}_{b}$. A shared preparation between Alice and Bob corresponds to a function $\omega$ on the pair of POVM elements such that $p\left(a, b \mid M_{A}, M_{B}\right)=\omega\left(Q_{a}, R_{b}\right)$. This, with no-signaling, implies that the marginal distributions are given by Born's rule, $p\left(a \mid M_{A}\right)=\operatorname{tr}\left(Q_{a} \rho_{A}\right)$ and $p\left(b \mid M_{B}\right)=\operatorname{tr}\left(R_{b} \rho_{B}\right)$, where $\rho_{A}$ and $\rho_{B}$ are quantum states (but the state of their joint system may not be quantum). We show that, in this setting, for any preparation $\omega$ there exist a joint quantum state $\sigma_{A B}$ and a relabeling of POVM measurements $\left\{\tilde{M}_{A}\right\}$ and $\left\{\tilde{M}_{B}\right\}$, such that $\omega\left(\tilde{Q}_{a}, R_{b}\right)=p\left(a, b \mid M_{A}, M_{B}\right)=$ $\operatorname{tr}\left(\left(\tilde{Q}_{a} \otimes \tilde{R}_{b}\right) \sigma_{A B}\right)$, where $\tilde{M}_{A}=\left\{\tilde{Q}_{a}\right\}_{a}$ and $\tilde{M}_{B}=\left\{\tilde{R}_{b}\right\}_{b}$. 
to information processing as those pointed out in [11]. Indeed, if we were able to surpass such bounds, then the local systems of Alice and Bob could not be quantum.

Other recent works also attempt to explain the limitations of quantum correlations. For example, the principle of information causality [13] starts with the assumption that nature demands that certain communication tasks should be hard to solve. Together with the assumption of the no-signaling principle, this allows one to obtain Tsirelson's bound for the special case of the CHSH inequality. In our work, we also assume the no-signaling principle, but combine it with a different assumption, namely, that the world is locally quantum; that is, quantum mechanics correctly describes the laws of nature of local physical systems. Making this assumption we recover the quantum limit on all possible nonlocal correlations (not only the Tsirelson's bound for the CHSH inequality).

Proof.-To prove our result, we now proceed in two steps. First, we explain a known characterization of all no-signaling probability assignments to local quantum measurements [16-18]. Second, we use this characterization to show that the resulting correlations can be obtained in quantum mechanics.

From local quantum measurements to positive on pure tensors (POPT) states.-Fix two finite dimensional Hilbert spaces on Alice and Bob's sides. A local quantum measurement (or POVM) consists of a pair of measurements $M_{A}$ and $M_{B}$ with outcome labels $\{a\}$ and $\{b\}$, respectively, on Alice's and Bob's Hilbert spaces. Such POVMs are described by complex Hermitian matrices $M_{A}=\left\{Q_{a}\right\}_{a}$, $M_{B}=\left\{R_{b}\right\}_{b}, Q_{a}, R_{b} \geq 0$, which sum to the identity, i.e., $\sum_{a} Q_{a}=\sum_{b} R_{b}=\mathbb{1}$ (see Fig. 1). A preparation shared between Alice and Bob assigns outcome probabilities $p\left(a, b \mid M_{A}, M_{B}\right)$ to any choice of measurements $M_{A}$ and $M_{b}$. More precisely, it corresponds to a function $\omega$ on the pair of POVM elements such that $p\left(a, b \mid M_{A}, M_{B}\right)=$ $\omega\left(Q_{a}, R_{b}\right)$.

Kläy et al. [17] have shown (see supplementary material [19]) that, assuming no-signaling, the shared preparations (or equivalently the functions $\omega$ ) are in one-to-one correspondence with matrices $W_{A B}$ such that $\operatorname{tr}\left(W_{A B}\right)=1$ and [20]

$$
p\left(a, b \mid M_{A}, M_{B}\right)=\operatorname{tr}\left(\left(Q_{a} \otimes R_{b}\right) W_{A B}\right) \geq 0 .
$$

The matrices $W_{A B}$ are called positive on pure tensors (POPT) states. All quantum states are POPT states, but there are POPT states that do not correspond to quantum states [21].

Note that POPT states cannot be combined arbitrarily [18]. For example, not all entangled measurements (measurements which are not a convex combination of tensor products $Q_{a} \otimes R_{b}$ ) of POPT states are well defined because they would result in negative "probabilities" for nonquantum POPTs. Specifically, if Alice and Bob share a POPT, and Charlie and Bob share another one, then if Alice and
Charlie come together, entangled measurements between their POPTs are not necessarily defined. This does not affect our result, since we are only interested in the case where we consider parties (here Alice and Charlie together) which are locally quantum.

From POPT states to quantum correlations.-We now show that there exist a quantum state $\sigma_{A B}$ and a map on POVM measurements

$$
f:\left\{M_{A}=\left\{Q_{a}\right\}_{a}\right\} \mapsto\left\{\tilde{M}_{A}=\left\{\tilde{Q}_{a}\right\}_{a}\right\},
$$

such that

$$
p\left(a, b \mid M_{A}, M_{B}\right)=\operatorname{tr}\left(\left(\tilde{Q}_{a} \otimes R_{b}\right) \sigma_{A B}\right) .
$$

In order to do so, we associate to each POPT state $W_{A B}$ a map $\mathcal{W}$ from matrices to matrices using the ChoiJamiołkowski isomorphism. Explicitly, $W_{A B}$ is obtained from $\mathcal{W}$ by acting on Bob's side of the (projection on the) maximally entangled state $|\Phi\rangle$

$$
W_{A B}=\mathbb{1} \otimes \mathcal{W}(|\Phi\rangle\langle\Phi|) .
$$

Because $W_{A B}$ is a POPT, the associated map $\mathcal{W}$ is positive; i.e., it sends positive matrices to positive ones, but it may not be an admissible quantum operation. Nevertheless, if $\mathcal{W}$ still maps POVMs to POVMs we can obtain the POPT correlations by moving the action of $\mathcal{W}$ from the maximally entangled state to the measurement elements. In particular, if $\mathcal{W}$ is unital $[\mathcal{W}(\mathbb{1})=\mathbb{1}]$, the map

$$
f: Q_{a} \mapsto \tilde{Q}_{a}=\mathcal{W}\left(Q_{a}^{T}\right)^{T}
$$

maps POVM measurements to POVM measurements. We then show that (7) holds with $\sigma_{A B}=|\Phi\rangle\langle\Phi|$. Let $d$ be the local dimension of Alice and Bob. If $\mathcal{W}$ is unital, we have

$$
\begin{aligned}
\operatorname{tr}\left(\left(Q_{a} \otimes R_{b}\right) W_{A B}\right) & =\operatorname{tr}\left(\left(Q_{a} \otimes R_{b}\right) \mathbb{1} \otimes \mathcal{W}(|\Phi\rangle\langle\Phi|)\right) \\
& =\operatorname{tr}\left(|\Phi\rangle\langle\Phi|\left(\mathbb{1} \otimes \mathcal{W}^{*}\right)\left(Q_{a} \otimes R_{b}\right)\right) \\
& =\operatorname{tr}\left(|\Phi\rangle\langle\Phi|\left(Q_{a} \otimes \mathcal{W}^{*}\left(R_{b}\right)\right)\right) \\
& =\frac{1}{d} \operatorname{tr}\left(Q_{a}^{T} \mathcal{W}^{*}\left(R_{b}\right)\right) \\
& =\frac{1}{d} \operatorname{tr}\left(\mathcal{W}\left(Q_{a}^{T}\right) R_{b}\right) \\
& =\operatorname{tr}\left(\left(\tilde{Q}_{a} \otimes R_{b}\right)|\Phi\rangle\langle\Phi|\right),
\end{aligned}
$$

where $\mathcal{W}^{*}$ denotes the adjoint of $\mathcal{W}$. This establishes (7) in the unital case.

In general, $\mathcal{W}$ can be decomposed into a unital map and another map. This other map gives a quantum state $\sigma_{A B}$ by acting on $|\Phi\rangle$. Then $f$ is defined in terms of the unital map as before. We finish the proof by showing that $\sigma_{A B}$ is well normalized and (7) is satisfied. For a general positive map, let $M$ be the image of the identity, i.e., $\mathcal{W}(\mathbb{1})=M$. The matrix $M$ is normalized, $\operatorname{tr}(M) / d=\operatorname{tr}\left(W_{A B}\right)=1$. We assume initially that $M$ is invertible, and define 


$$
\tilde{\mathcal{W}}(\cdot)=M^{-1 / 2} \mathcal{W}(\cdot) M^{-1 / 2} .
$$

The map $\tilde{\mathcal{W}}$ is unital. Further, the quantum state $\sigma_{A B}=$ $|\psi\rangle\langle\psi|$ given by

$$
|\psi\rangle=\left(M^{1 / 2}\right)^{T} \otimes \mathbb{1} \Phi
$$

is well normalized; that is, $\operatorname{tr}\left(\sigma_{A B}\right)=\operatorname{tr}\left(M^{T}\right) / d=1$. Thus by defining $f$ as in (9) but in terms of $\tilde{\mathcal{W}}$, we conclude

$$
\begin{aligned}
\operatorname{tr}\left(\left(Q_{a} \otimes R_{b}\right) W_{A B}\right) & =\frac{1}{d} \operatorname{tr}\left(\mathcal{W}\left(Q_{a}^{T}\right) R_{b}\right) \\
& =\frac{1}{d} \operatorname{tr}\left(\left(M^{1 / 2} \tilde{\mathcal{W}}\left(Q_{a}^{T}\right) M^{1 / 2}\right) R_{b}\right) \\
& =\operatorname{tr}\left(\left(\tilde{Q}_{a} \otimes R_{b}\right) \sigma_{A B}\right) .
\end{aligned}
$$

If $M$ is not invertible, in order to define $\tilde{\mathcal{W}}$, one can start with the map $(1-\epsilon) \mathcal{W}(\cdot)+\epsilon \mathbb{1} \operatorname{tr}(\cdot)$, and then take the limit $\epsilon \rightarrow 0$.

Conclusion.-We have shown that being locally quantum is sufficient to ensure that all nonlocal correlations between distant parties can be reproduced quantum mechanically, if the principle of no-signaling is obeyed. This gives us a natural explanation of why quantum correlations are weaker than is required by the no-signaling principle alone; i.e., given that one can describe local physics according to quantum measurements and states, then nosignaling already implies quantum correlations.

It would be interesting to know whether our work can be used to derive more efficient tests for nonlocal quantum correlations than those proposed in [14]. Finally, it is an intriguing question whether one can find new limits on our ability to perform information processing locally based on the limits of nonlocal correlations, which we now know to demand local quantum behavior.

This work was supported by the National Science Foundation under Grant No. PHY-0803371 through the Institute for Quantum Information at the California Institute of Technology, and by the U.S. Department of Energy through the LDRD program at Los Alamos National Laboratory. Research at Perimeter Institute is supported by the Government of Canada through Industry Canada and by the Province of Ontario through the Ministry of Research and Innovation.

*boixo@caltech.edu

[1] E. Schrödinger, Proc. Cambridge Philos. Soc. 31, 555 (1935).

[2] W. Tittel et al., Phys. Rev. Lett. 81, 3563 (1998); G. Weihs et al., Phys. Rev. Lett. 81, 5039 (1998); J. Pan et al.,
Nature (London) 403, 515 (2000); M. A. Rowe et al., Nature (London) 409, 791 (2001); M. Ansmann et al., Nature (London) 461, 504 (2009).

[3] J. S. Bell, Physics (Long Island City, N.Y.) 1, 195 (1965).

[4] B. Julsgaard, A. Kozhekin, and E.S. Polzik, Nature (London) 413, 400 (2001); L. Duan et al., Nature (London) 414, 413 (2001); H. Haffner et al., Nature (London) 438, 643 (2005); C. W. Chou et al., Nature (London) 438, 828 (2005); D. L. Moehring et al., Nature (London) 449, 68 (2007).

[5] S. Popescu and D. Rohrlich, Found. Phys. 24, 379 (1994).

[6] J. Clauser et al., Phys. Rev. Lett. 23, 880 (1969).

[7] B. Tsirelson, Lett. Math. Phys. 4, 93 (1980).

[8] S. Popescu, Nature Phys. 2, 507 (2006).

[9] A. Leggett, Found. Phys. 33, 1469 (2003); S. Groblacher et al., Nature (London) 446, 871 (2007); C. Branciard et al., Nature Phys. 4, 681 (2008).

[10] S. Wolf and J. Wullschleger arXiv:quant-ph/0508233; G. Brassard et al., Phys. Rev. Lett. 96, 250401 (2006); H. Buhrman et al., Proc. R. Soc. A 462, 1919 (2006); A.J. Short, N. Gisin, and S. Popescu, Quant. Info. Proc. 5, 131 (2006); H. Barnum et al., Phys. Rev. Lett. 99, 240501 (2007); H. Barnum et al., in Proceedings of the IEEE Information Theory Workshop (IEEE, Piscataway, NJ, 2008), pp. 386-390; E. Hänggi, R. Renner, and S. Wolf, arXiv:0906.4760.

[11] W. van Dam, arXiv:quant-ph/0501159.

[12] G. V. Steeg and S. Wehner, Quantum Inf. Comput. 9, 801 (2009).

[13] M. Pawlowski et al., Nature (London) 461, 1101 (2009).

[14] S. Wehner, Phys. Rev. A 73, 022110 (2006); M. Navascués, S. Pironio, and A. Acin, New J. Phys. 10, 073013 (2008); A. Doherty et al., in Proceedings of the 23rd IEEE Conference on Computational Complexity (IEEE, Piscataway, NJ, 2008), pp. 199-210.

[15] Which also holds for dimension $d=2$. A. Gleason, J. Math. Mech. 6, 885 (1957); P. Busch, Phys. Rev. Lett. 91, 120403 (2003); C. Caves et al., Found. Phys. 34, 193 (2004).

[16] D. Foulis and C. Randall, in Interpretations and Foundations of Quantum Theory, edited by H. Neumann (Bibliographisches Institut Wissenschaftverlag, Mannheim, 1980), Vol. 5, pp. 9-20.

[17] M. Kläy, C. Randall, and D. Foulis, Int. J. Theor. Phys. 26, 199 (1987).

[18] H. Barnum et al., arXiv:quant-ph/0507108.

[19] See supplementary material at http://link.aps.org/ supplemental/10.1103/PhysRevLett.104.140401 for details on the matrix representation of POPT states.

[20] In the same reference it is shown that classical communication in the performance of measurements rules out signaling in the direction opposite of the classical communication.

[21] Note that our result does not rule out the existence of POPT states, but shows that they do not generate stronger than quantum correlations. 